\title{
Comparing hemostatic resuscitation management of intraoperative massive bleeding with traumatic massive bleeding: a computer simulation
}

\section{Young Sun Lee ${ }^{1}$, Kyu Nam Kim², Min Kyu Lee², Jung Eun Sun², Hyun Jin Lim², and Jong Hun Jun ${ }^{2}$}

${ }^{1}$ Department of Medicine, Hanyang University Graduate School, ${ }^{2}$ Department of Anesthesiology and Pain Medicine, Hanyang University College of Medicine, Seoul, Korea
Received May 24, 2020

Revised September 9, 2020

Accepted September 14, 2020

\section{Corresponding author}

Jong Hun Jun, M.D., Ph.D.

Department of Anesthesiology and Pain Medicine, Hanyang University College of Medicine, 222 Wangsimniro, Seongdong-gu, Seoul 04763, Korea

Tel: 82-2-2290-8680

Fax: 82-2-2299-0742

E-mail: jhjun@hanyang.ac.kr
Background: Appropriate blood component transfusion might differ between intraoperative massive bleeding and traumatic massive bleeding in the emergency department because trauma patients initially bleed undiluted blood and replacement typically lags behind blood loss. We compared these two blood loss scenarios, intraoperative and traumatic, using a computer simulation.

Methods: We modified the multi-compartment dynamic model developed by Hirshberg and implemented it using STELLA 9.0. In this model, blood pressure changes as blood volume fluctuates as bleeding rate and transcapillary refill rate are controlled by blood pressure. Using this simulation, we compared the intraoperative bleeding scenario with the traumatic bleeding scenario. In both scenarios, patients started to bleed at a rate of $50 \mathrm{ml} / \mathrm{min}$. In the intraoperative bleeding scenario, fluid was administered to maintain isovolemic status; however, in the traumatic bleeding scenario, no fluid was supplied for up to $30 \mathrm{~min}$ and no blood was supplied for up to 50 min. Each unit of packed red blood cells (PRBC) was given when the hematocrit decreased to $27 \%$, fresh frozen plasma (FFP) was transfused when plasma was diluted to $30 \%$, and platelet concentrate (PC) was transfused when platelet count became 50,000/ml.

Results: In both scenarios, the appropriate ratio of PRBC:FFP was 1:0.47 before PC transfusion, and the ratio of PRBC:FFP:platelets was 1:0.35:0.39 after initiation of PC transfusion.

Conclusion: The ratio of transfused blood component did not differ between the intraoperative bleeding and traumatic bleeding scenarios.

Keywords: Blood coagulation disorder; Blood component transfusion; Computer simulation; Hemorrhage.

\section{INTRODUCTION}

Persistent hemorrhage remains a major contributor to mortality in massively transfused patients, many of whom develop coagulopathy [1]. Coagulopathy is a component of the lethal triad of death that also includes hypothermia

This is an Open Access article distributed under the terms of the Creative Commons Attribution Non-Commercial License (http://creativecommons.org/licenses/by-nc/4.0) which permits unrestricted non-commercial use, distribution, and reproduction in any medium, provided the original work is properly cited.

Copyright (C) the Korean Society of Anesthesiologists, 2020 
and acidosis [2-4]. Coagulopathy-related diffuse bleeding is difficult to manage. The causes of coagulopathy are multifactorial and interrelated, including consumption and dilution of coagulation factors and platelets, dysfunction of platelets and the coagulation system, increased fibrinolysis, compromise of coagulation by the infusion of colloids, hypocalcemia, and disseminated intravascular coagulation-like syndrome [5-7].

Although the extreme circumstances of massive hemorrhage do not allow prospective controlled trials, computer modeling offers an interesting alternative. A computer simulation can capture the interactions between bleeding, hemodynamics, hemodilution, and replacement as they unfold during severe hemorrhage. Various replacement options can then be applied to the model to evaluate effectiveness in preventing or correcting dilutional coagulopathy [8].

In this study, we focused on dilutional coagulopathy in both intraoperative bleeding and traumatic bleeding scenarios. Prior to the era of blood component transfusion, the transfusion of large volumes of stored blank blood did not result in hemorrhagic diathesis in young and previously healthy soldiers wounded during the Vietnam war [9]. However, recent resuscitation with crystalloids, colloids, and/or packed red blood cells (PRBCs) can result in dilutional coagulopathy. Because trauma patients bleed undiluted blood initially and replacement typically lags behind blood loss, it was hypothesized that there would be differences between patients who bleed during surgery and patients who receive emergent resuscitation after a period of traumatic massive bleeding. Furthermore, in trauma patients, shock, tissue hypoxia, acidosis, and hypothermia can aggravate bleeding tendency.

To date, no consensus has been reached regarding what, when, how much fluid, and what blood component should be given during massive transfusion [10]. Appropriate blood component therapy during hemostatic resuscitation might be different between intraoperative bleeding and traumatic bleeding. We therefore compared when and how much of each blood component should be given in both of these scenarios using computer simulation.

\section{MATERIALS AND METHODS}

We modified the multi-compartment dynamic model developed by Hirshberg [8] and implemented it using a graphical modeling tool STELLA 9.0 (High Performance
Systems, USA). In this model, blood pressure changed as blood volume fluctuated, and bleeding rate and transcapillary refill rate were controlled by blood pressure. With ongoing bleeding and transfusion, hematocrit and dilution of clotting factors and platelets were calculated.

Blood volume consists of three compartments: red cells, plasma, and virtual intravascular water. Intravascular water accepts crystalloid infusion and exchanges free water with interstitial spaces (Fig. 1). Equations for the fraction of infused crystalloids that is retained in circulation during massive hemorrhage [11], transcapillary refill rate [12-14], and the pressure-volume relationship of circulation $[12,15]$ are the same as in the Hirshberg model. Fibrinogen dilution was assumed to be directly proportional to hemodilution $[7,9]$. Correcting function was used in calculating platelet level [8]. The entire set of model equations is given in the Supplementary Materials.

\section{Blood components}

Information on blood components was obtained from the Korea Center for Disease Control and Prevention 2013 transfusion guidelines (Table 1). The average volume of PRBCs derived from $400 \mathrm{ml}$ of whole blood was $243.12 \pm$

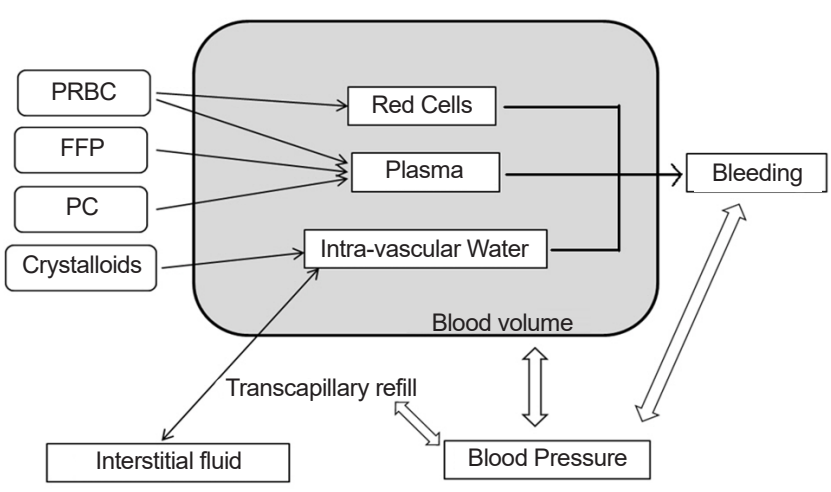

Fig. 1. Overview of the model. Arrows represent the mathematical relationships between compartments. PRBC: packed red blood cells, FFP: fresh frozen plasma, PC: platelet concentrate.

Table 1. Blood Compartment Information in the Transfusion Guidelines, Korea Center for Disease Control and Prevention, 2013

\begin{tabular}{lccc}
\hline & PRBC & FFP & Platelets \\
\hline Volume $(\mathrm{ml})$ & $243.12 \pm 13.48$ & $155.03 \pm 12.11$ & $50.85 \pm 1.90$ \\
Hematocrit $(\%)$ & $70.31 \pm 4.16$ & & \\
Count $\left(\times 10^{9} /\right.$ unit) & & & $66.7 \pm 11.7$ \\
\hline
\end{tabular}

Values are presented as mean \pm SD. PRBC: packed red blood cells, FFP: fresh frozen plasma. 
$13.48 \mathrm{ml}$. Hematocrit was $70.31 \%$ on average. As a result, red blood cell volume was at an average of $170 \mathrm{ml}$. We assumed all PRBCs were derived from $400 \mathrm{ml}$ of whole blood for convenience. In the manufacturing blood compartment, $56 \mathrm{ml}$ of citrate-phosphate-dextrose with adenine (CPDA-1) solution was added. Plasma volume in PRBCs was $243-170=73 \mathrm{ml}$, which includes some amount of CPDA-1 solution. Total plasma volume in whole blood including CPDA-1 solution was $73+155+51=279 \mathrm{ml}$. Volume of CPDA-1 solution in PRBCs was $73 \times 56 / 279=15$ ml. Calculated real plasma volume contained in PRBCs was $73-15=58 \mathrm{ml}$, which excluded the volume of CPDA1 solution.

The average volume of plasma derived from $400 \mathrm{ml}$ whole blood was $155.03 \pm 12.11 \mathrm{ml}$. Except for the volume of CPDA-1 solution, each fresh frozen plasma (FFP) was assumed to contain $124 \mathrm{ml}$ of plasma. The average volume of platelet concentrate (PC) derived from $400 \mathrm{ml}$ whole blood was $50.85 \pm 1.90 \mathrm{ml}$. Except for the volume of CPDA1 solution, each unit of PC contained $41 \mathrm{ml}$ of plasma and $6.67 \times 10^{10}$ platelets on average, of which one third underwent splenic sequestration when transfused [16].

\section{The reference scenario}

We generated an intraoperative bleeding scenario and a trauma bleeding scenario. Both scenarios included a $70 \mathrm{~kg}$ person whose blood volume, hematocrit, fibrinogen concentration, and platelets of $4.9 \mathrm{~L}, 42 \%, 3 \mathrm{~g} / \mathrm{L}$, and 230,000/ $\mathrm{ml}$ was assumed, respectively. In both scenarios, patients started to bleed at a rate of $50 \mathrm{ml} / \mathrm{min}$.

The intraoperative bleeding scenario was based on a washout equation by designing the normovolemic status to be maintained by administering fluid. As bleeding continued, the blood pressure was assumed to be unchanged. If hematocrit decreased to $27 \%$, one unit of PRBCs was given for $10 \mathrm{~min}$. FFP was transfused for $10 \mathrm{~min}$ when plasma was diluted to $30 \%$ of the original plasma. Each unit of PC was transfused for $5 \mathrm{~min}$ when platelet count became $50,000 / \mathrm{ml}$. FFP and PC starting time followed the practice guidelines for perioperative blood transfusion and adjuvant therapies [17].

In the traumatic bleeding scenario, the patient was set to bleed for 30 min without fluid supply until arrival at the emergency room (ER). The traumatic bleeding scenario was not only based on a washout equation, but also adopted a pressure-volume relationship concept. Using the pres- sure-volume equation, the amount of bleeding per minute reduced as blood pressure decreased due to a loss of blood. From arrival in the ER, crystalloid was administered via an intravenous line at the rate of $300 \mathrm{ml} / \mathrm{min}$ until the patient recovered to isovolemic status. After $50 \mathrm{~min}$ of trauma, blood components were prepared and each unit of PRBCs was given when hematocrit decreased to $27 \%$. FFP was transfused when plasma was diluted to $30 \%$, and PC was transfused when platelet count became $50,000 / \mathrm{ml}$. The infusion of each blood component was same as in the intraoperative bleeding scenario.

\section{RESULTS}

In the intraoperative bleeding scenario (Fig. 2), bleeding during operation began at time 0 and PRBC administration started at $43 \mathrm{~min}$ when the bleeding volume was 0.44 times total blood volume (Table 2). In the traumatic bleeding scenario (Fig. 3), the patient lost 1,175 $\mathrm{ml}$ of blood volume and transcapillary refill volume was $449 \mathrm{ml}$ until arrival in the ER when the systolic blood pressure was $84 \mathrm{mmHg}$. PRBC administration was started at 52 min when bleeding volume was 0.42 times total blood volume. In the intraoperative bleeding scenario, FFP administration started at 164 min when bleeding volume was 1.67 times the total blood volume. In the traumatic bleeding scenario, FFP administration started at $170 \mathrm{~min}$ when bleeding volume was 1.63 times the total blood volume. During FFP transfusion, the appropriate PRBC:FFP ratio was 1:0.47 in both scenarios.

PC administration was started at 219 min when bleeding volume was 2.24 times the total blood volume in the intraoperative bleeding scenario and at $225 \mathrm{~min}$ when bleeding volume was 2.20 times the total blood volume in the traumatic bleeding scenario. After the start of PC transfusion, the appropriate PRBC:FFP:PC ratio was 1:0.35:0.39 in both scenarios.

\section{DISCUSSION}

Computer simulations regarding dilutional coagulopathy have been previously studied $[8,9,16]$. Here, we designed two separate scenarios and compared them. In the traumatic bleeding scenario, the amount of bleeding per minute reduced by decreased blood pressure compared to intraoperative scenario; as a consequence, the onset of dilutional coagulopathy was delayed. Similar results have been 


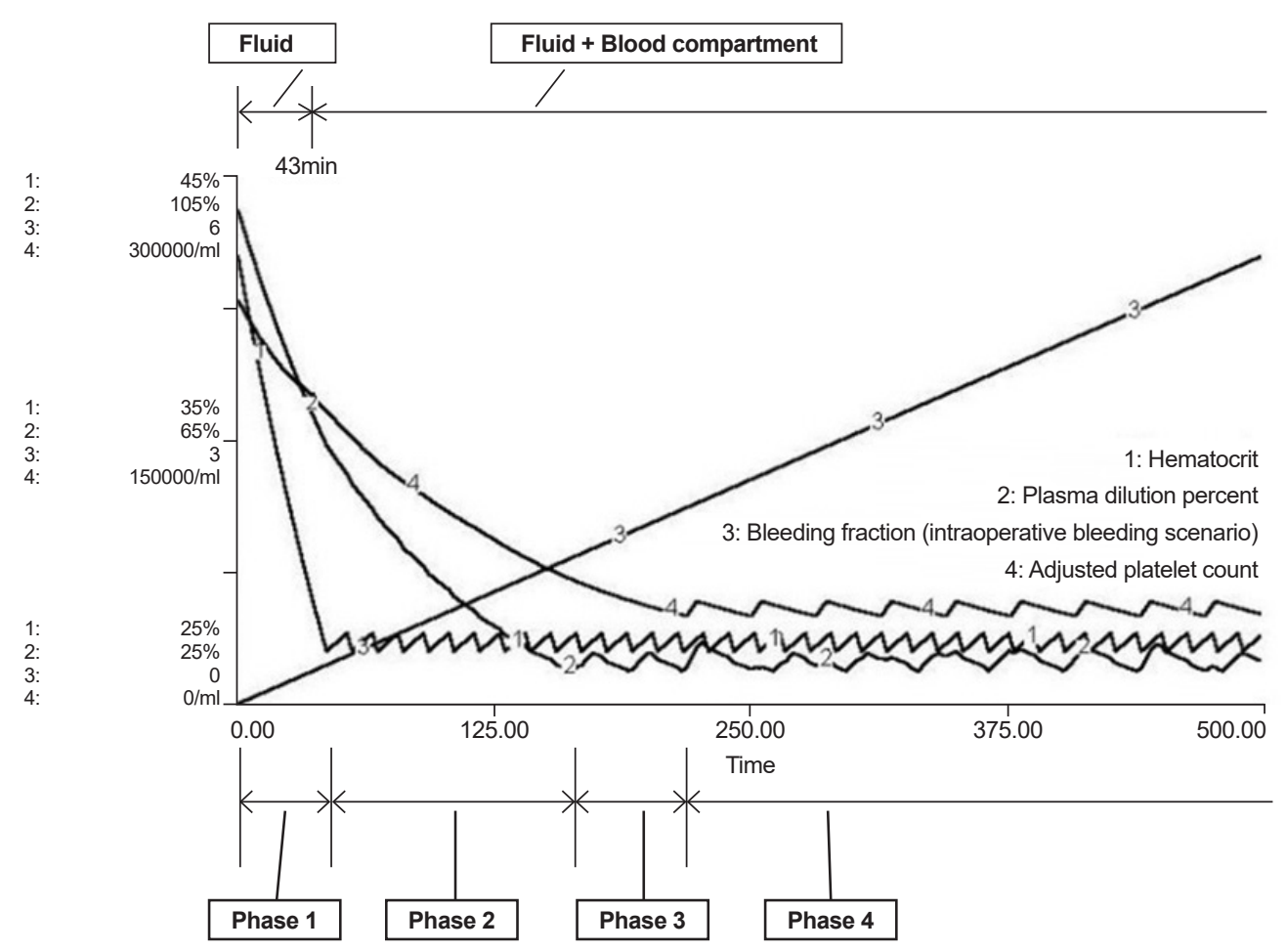

Fig. 2. Model prediction of hematocrit (\%), plasma dilution (\%), bleeding fraction and adjusted platelet count (/ml) in the intraoperative bleeding scenario. Phase 1: bleeding started, but only crystalloid fluid was given to maintain normal blood pressure. Phase 2: PRBC administration was started at $43 \mathrm{~min}$ and the bleeding fraction was 0.44. PRBCs were administered. Phase 3: FFP was started at 164 min and the bleeding fraction was1.67. PRBCs and FFP were given. Phase 4: PC was started at 219 min and the bleeding fraction was 2.24. PRBCs, FFP, and PC were given. Bleeding fraction: cumulative blood loss / initial blood volume. PRBC: packed red blood cells, FFP: fresh frozen plasma, PC: platelet concentrate.

Table 2. The Outcome of Two Scenarios

\begin{tabular}{lcc}
\hline & Intraoperative bleeding scenario & Traumatic bleeding scenario \\
\hline PRBC:FFP & $1: 0.47$ & $1: 0.47$ \\
PRBC:FFP:PC & $1: 0.35: 0.39$ & $1: 0.35: 0.39$ \\
Time of PRBC administration after initiation of bleeding ( $\mathrm{min})$ & 43 & 52 \\
Time of FFP administration after initiation of bleeding $(\mathrm{min})$ & 164 & 170 \\
Time of PC administration after initiation of bleeding $(\mathrm{min})$ & 219 & 225 \\
Bleeding fraction at initiation of PRBC transfusion & 0.44 & 0.42 \\
Bleeding fraction at initiation of FFP transfusion & 1.67 & 1.63 \\
Bleeding fraction at initiation of PC transfusion & 2.24 & 2.2
\end{tabular}

PRBC: packed red blood cells, FFP: fresh frozen plasma, PC: platelet concentrate. Bleeding fraction: cumulative blood loss / initial blood volume.

shown by Hirshberg et al. $[8,18]$.

The key Hirshberg model equations and parameters were not complete as is, and did not work when entered into the STELLA program. Therefore, we modified some parts of the equation to implement the model and attempt to reproduce the results of the Hirshberg study. As such, our results have some differences when compared with Hirshberg, which suggest two options for giving FFP before the prothrombin time (PT) crossover time. PT crossover time means the point during a simulation when a clotting test first crosses its respective threshold. In other words, the time when the plasma fraction goes below $30 \%$ of the original plasma fraction. The first option is to use an aggressive lower PRBC/FFP replacement ratio such as 3:2 and the second option is to give two units of FFP concurrently with the first units of PRBCs at the beginning of the operation. This suggestion by Hirshberg et al. $[8,18]$ is a much lower PRBC/FFP ratio than the 5:2 to 5:3 ratio used in many massive transfusion protocols and also much lower than our simulation results of $1: 0.47=5: 2.35$ ratio 


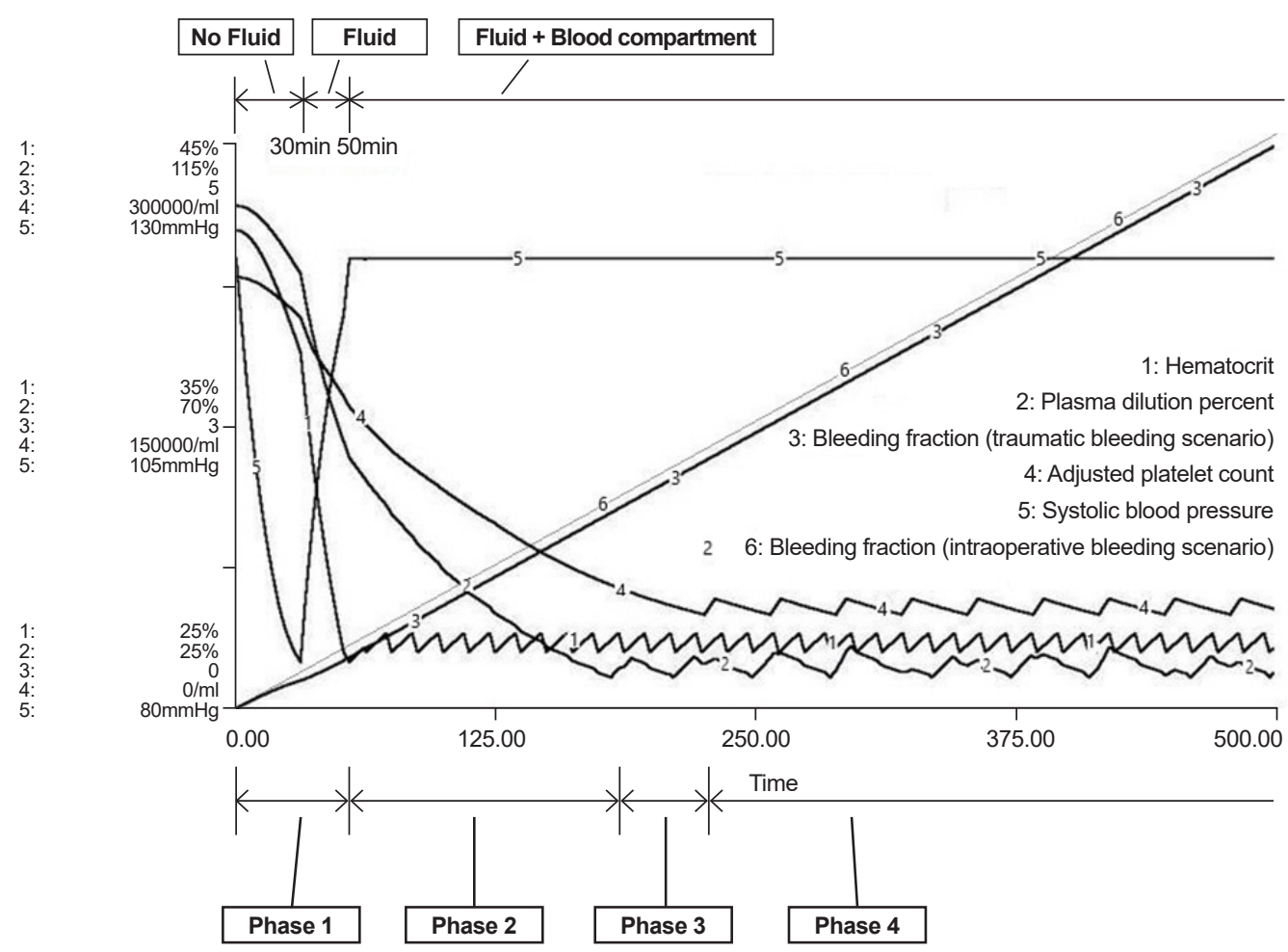

Fig. 3. Model prediction of hematocrit (\%), plasma dilution (\%), bleeding fraction, adjusted platelet count ( $/ \mathrm{ml}$ ) and systolic blood pressure (mmHg) in the traumatic bleeding scenario. Phase 1: bleeding started and no fluid was administered until 30 min when the patient arrived at the ER. At that time, blood pressure was $84 \mathrm{mmHg}$, bleeding volume was $1,175 \mathrm{ml}$, and transcapillary refill was $449 \mathrm{ml}$. Administration of crystalloid fluid started. Phase 2: PRBC administration was started at 52 min and the bleeding fraction was 0.42. Only PRBCs were given. Phase 3: FFP administration was started at 170 min and the bleeding fraction was 1.63. PRBCs and FFP were given. Phase 4: PC administration was started at 225 min and the bleeding fraction was 2.20. PRBCs, FFP, and PC were given. Bleeding fraction: cumulative blood loss / initial blood volume. ER: emergency room, PRBC: packed red blood cells, FFP: fresh frozen plasma, PC: platelet concentrate.

\section{$[17,19]$.}

In the Hirschberg model, patient initial bleeding rate was $135 \mathrm{ml} / \mathrm{min}$ with $3,297 \mathrm{ml}$ lost and $67 \%$ of the estimated blood volume at the beginning of the operation. However, in our traumatic bleeding scenario, initial bleeding rate was $50 \mathrm{ml} / \mathrm{min}$ with $1,175 \mathrm{ml}$ lost and $41 \%$ of the estimated blood volume, and FFP transfusion was started during the 11th PRBC transfusion. We think the reason why this difference occurred was because the increased massive bleeding without hemodilution incurred a greater loss of clotting factor. Comparing our intraoperative bleeding scenario (FFP transfusion started at 1.67 blood volume loss) with the trauma scenario (FFP transfusion started at 1.63 blood volume loss), dilutional coagulopathy began at less blood loss. Early FFP transfusion in the Hirshberg model could be due to early massive blood loss in that scenario [8].

Hirshberg ignored plasma contained in PRBCs and PC. PRBCs contain a small amount of plasma (30-60 ml) as does PC (approximately $80 \mathrm{ml}$ ) [20]. In our model, we assumed PRBCs contain $58 \mathrm{ml}$ of plasma and PC contains 41 $\mathrm{ml}$ of plasma, both without CPDA-1 solution, by using information of the blood compartment obtained in the 2013 transfusion guidelines. These are not negligible volumes compared with $124 \mathrm{ml}$ of plasma contained in FFP without CPDA-1 solution. If plasma contained PRBCs and PC is not considered, the calculated PRBC/FFP ratio will decrease. Therefore, the Hirshberg 3:2 PRBC/FFP ratio could be underestimated.

The Pragmatic, Randomized Optimal Platelet and Plasma Ratios trial was designed to address the effectiveness and safety of transfusing PRBC, FFP, PLT in a 1:1:1 ratio compared with a 2:1:1 ratio in patients with trauma who were predicted to receive a massive transfusion. Among patients with severe trauma and major bleeding, early administration of PRBC, FFP, PLT in a 1:1:1 ratio compared with a 2:1:1 ratio did not result in significant differences in mortality at $24 \mathrm{~h}$ or 30 days. However, more patients in the 1:1:1 group achieved hemostasis and fewer experienced death due to exsanguination by $24 \mathrm{~h}$. Even though there was an increased use of FFP and PLT transfused in the 1:1:1 
group, no other safety differences were identified between the two groups [21]. Conversely, other studies have reported beneficial outcomes across a wider range of blood product ratios or goal-directed approaches [22,23].

Our simulation shows a 1:0.47 ratio for PRBC:FFP and a 1:0.35:0.39 ratio for PRBC:FFP:PC, if dilutional coagulopathy is assumed to occur when plasma dilution is $<30 \%$, fibrinogen is $<0.8 \mathrm{~g} / \mathrm{L}$, and platelets are $<50,000 / \mathrm{ml}$. Interpreting this result requires circumspection; however, patients generally have a wide range of coagulation problems such as dysfunction of platelets and the coagulation system, increased fibrinolysis, compromise of coagulation by the infusion of colloids, hypocalcemia, disseminated intravascular coagulation-like syndrome, acidosis, hyperthermia, and the destruction of clotting factor in stored FFP $[24,25]$. In the massive transfusion scenario, avoiding the above listed problems is not easy and computer simulations will not perfectly imitate the conditions of the real body. So, our results can be used only as reference and not indication. Careful observation of operation in the field, communication with surgeons, and repetitive coagulation tests are the most important variables in preventing coagulopathy.

The results of this simulation show that coagulopathy can occur at less blood loss due to the loss of undiluted blood if fluids are not infused after the start of bleeding. In addition, it must be noted that the coagulopathy and transfusion start times were delayed because low blood pressure reduces hemorrhage. Restricting fluid infusion seems to delay dilutional coagulopathy but it can generate circulation problems and can bring about more disastrous consequences to the patient. Therefore, restricting fluid technique to delay reduction of hemorrhage and dilutional coagulopathy must be done cautiously. In addition, the difference in the time when coagulopathy occurred between the two scenarios was negligible in our study. More than likely, the amount of hemorrhage (about $20 \%$ of total blood volume) was too small to make a difference and transcapillary refill could have attributed to make the difference even less negligible.

Massive transfusion protocols are widely various among hospitals, with replacement ratios ranging between 10:1 and 5:3 for PRBC:FFP and between 10:6 and 10:12 for PRBC:PLT $[26,27]$. Clinical suitability and justification of the various massive transfusion practices in trauma or operations is clearly required. Due to the limitations of computer modeling when compared with a biologic system or clini- cal trial data, our findings surely need validation and further assessment. However, computer simulation models can uncover new predictions and generate data that can be used in place of harmful clinical trials that would be against medical ethics; as such, increased need for computer simulations in clinical experiments is apparent.

In conclusion, according to computer simulation, the appropriate blood component ratio might be a 1:0.47 ratio for PRBC:FFP and a 1:0.35:0.39 ratio for PRBC:FFP:PC, which did not differ between the intraoperative bleeding and traumatic bleeding scenarios.

\section{SUPPLEMENTARY MATERIALS}

Supplementary data including key model equations and parameters can be found online at https://doi.org/10. 17085/apm.20042.

\section{CONFLICTS OF INTEREST}

No potential conflict of interest relevant to this article was reported.

\section{AUTHOR CONTRIBUTIONS}

Conceptualization: Young Sun Lee, Kyu Nam Kim, Hyun Jin Lim, Jong Hun Jun. Methodology: Young Sun Lee. Writing -original draft: Young Sun Lee, Jong Hun Jun. Writing review \& editing: Young Sun Lee, Kyu Nam Kim, Min Kyu Lee, Jung Eun Sun, Hyun Jin Lim. Investigation: Young Sun Lee, Jong Hun Jun. Software: Young Sun Lee. Supervision: Jong Hun Jun.

\section{ORCID}

Young Sun Lee, https://orcid.org/0000-0002-9362-5493 Kyu Nam Kim, https://orcid.org/0000-0003-2526-8348

Min Kyu Lee, https://orcid.org/0000-0003-2025-7244

Jung Eun Sun, https://orcid.org/0000-0002-1023-9037 Hyun Jin Lim, https://orcid.org/0000-0002-2040-8826 Jong Hun Jun, https://orcid.org/0000-0003-3579-1765

\section{REFERENCES}

1. Guerado E, Medina A, Mata MI, Galvan JM, Bertrand ML. Protocols for massive blood transfusion: when and why, and potential complications. Eur J Trauma Emerg Surg 2016; 42: 283- 
95.

2. Gentilello LM, Pierson DJ. Trauma critical care. Am J Respir Crit Care Med 2001; 163(3 Pt 1): 604-7.

3. Cinat ME, Wallace WC, Nastanski F, West J, Sloan S, Ocariz J, et al. Improved survival following massive transfusion in patients who have undergone trauma. Arch Surg 1999; 134: 964-8; discussion 968-70.

4. Eddy VA, Morris JA Jr, Cullinane DC. Hypothermia, coagulopathy, and acidosis. Surg Clin North Am 2000; 80: 845-54.

5. Hardaway RM. The significance of coagulative and thrombotic changes after haemorrhage and injury. J Clin Pathol Suppl (R Coll Pathol) 1970; 4: 110-20.

6. Miller RD, Robbins TO, Tong MJ, Barton SL. Coagulation defects associated with massive blood transfusions. Ann Surg 1971; 174: 794-801.

7. Reiss RF. Hemostatic defects in massive transfusion: rapid diagnosis and management. Am J Crit Care 2000 9: 158-65; quiz 166-7.

8. Hirshberg A, Dugas M, Banez EI, Scott BG, Wall MJ Jr, Mattox KL. Minimizing dilutional coagulopathy in exsanguinating hemorrhage: a computer simulation. J Trauma 2003; 54: 45463.

9. Hiippala S. Replacement of massive blood loss. Vox Sang 1998; 74 Suppl 2: 399-407.

10. Flint AWJ, McQuilten ZK, Wood EM. Massive transfusions for critical bleeding: is everything old new again? Transfus Med 2018; 28: 140-9.

11. Cervera AL, Moss G. Crystalloid distribution following hemorrhage and hemodilution: mathematical model and prediction of optimum volumes for equilibration at normovolemia. J Trauma 1974; 14: 506-20.

12. Lewis FR Jr. Prehospital intravenous fluid therapy: physiologic computer modelling. J Trauma 1986; 26: 804-11.

13. Mardel SN, Simpson SH, Kelly S, Wytch R, Beattie TF, Menezes G. Validation of a computer model of haemorrhage and transcapillary refill. Med Eng Phys 1995; 17: 215-8.

14. Simpson SH, Menezes G, Mardel SN, Kelly S, White R, Beattie T. A computer model of major haemorrhage and resuscitation. Med Eng Phys 1996; 18: 339-43.

15. Wears RL, Winton CN. Load and go versus stay and play: analysis of prehospital i.v. fluid therapy by computer simulation. Ann Emerg Med 1990; 19: 163-8.

16. Lundsgaard-Hansen P. Treatment of acute blood loss. Vox Sang
1992; 63: 241-6.

17. American Society of Anesthesiologists Task Force on Perioperative Blood Transfusion and Adjuvant Therapies. Practice guidelines for perioperative blood transfusion and adjuvant therapies: an updated report by the American Society of Anesthesiologists Task Force on Perioperative Blood Transfusion and Adjuvant Therapies. Anesthesiology 2006; 105: 198-208.

18. Hirshberg A, Hoyt DB, Mattox KL. Timing of fluid resuscitation shapes the hemodynamic response to uncontrolled hemorrhage: analysis using dynamic modeling. J Trauma 2006; 60: 1221-7.

19. Spahn DR, Bouillon B, Cerny V, Coats TJ, Duranteau J, Fernández-Mondéjar E, et al. Management of bleeding and coagulopathy following major trauma: an updated European guideline. Crit Care 2013; 17: R76.

20. Hardy JF, De Moerloose P, Samama M. Massive transfusion and coagulopathy: pathophysiology and implications for clinical management. Can J Anaesth 2004; 51: 293-310.

21. Holcomb JB, Tilley BC, Baraniuk S, Fox EE, Wade CE, Podbielski JM, et al. PROPPR Study Group. Transfusion of plasma, platelets, and red blood cells in a 1:1:1 vs a 1:1:2 ratio and mortality in patients with severe trauma: the PROPPR randomized clinical trial. JAMA 2015; 313: 471-82.

22. Johnson JL, Moore EE, Kashuk JL, Banerjee A, Cothren CC, Biffl WL, et al. Effect of blood products transfusion on the development of postinjury multiple organ failure. Arch Surg 2010; 145: 973-7.

23. Scalea TM, Bochicchio KM, Lumpkins K, Hess JR, Dutton R, Pyle A, et al. Early aggressive use of fresh frozen plasma does not improve outcome in critically injured trauma patients. Ann Surg 2008; 248: 578-84.

24. Scott E, Puca K, Heraly J, Gottschall J, Friedman K. Evaluation and comparison of coagulation factor activity in fresh-frozen plasma and 24-hour plasma at thaw and after 120 hours of 1 to $6^{\circ} \mathrm{C}$ storage. Transfusion 2009; 49: 1584-91.

25. Spahn DR, Rossaint R. Coagulopathy and blood component transfusion in trauma. Br J Anaesth 2005; 95: 130-9.

26. Johnson JW, Gracias VH, Schwab CW, Reilly PM, Kauder DR, Shapiro MB, et al. Evolution in damage control for exsanguinating penetrating abdominal injury. J Trauma 2001; 51: 261-9; discussion 269-71.

27. Robb WJ. Massive transfusion in trauma. AACN Clin Issues 1999; 10: 69-84; quiz 138-40. 